Pacific Journal of Mathematic 


\section{THE POLYNOMIAL HULL OF A THIN TWO-MANIFOLD}

\section{Michael FREEMAN}

Let $M$ be a smooth real two dimensional submanifold of $C^{2}$. Near a point of $M$ where its tangent space is complex linear it may, after a certain local biholomorphic change of coordinates, be represented as the graph near 0 in $C^{2}$ of a smooth function

$$
f(z)=Q(z)+o\left(|z|^{2}\right)
$$

in which $z$ is the first coordinate of $C^{2}$ and $Q$ is a real valued quadratic form. This paper is concerned with the polynomially convex hull of a small compact set $K$ in $M$ near 0 and associated descriptions of the Banach algebra $P(M)$ of continuous functions uniformly approximable on $K$ by polynomials in two complex variables. It treats the very special case where $f$ has rank $\leqq 1$ near 0 (as an $\boldsymbol{R}^{2}$-valued map). It is shown that if $Q$ has nonzero eigenvalues of opposite sign, then all sufficiently small compact sets $K$ are polynomially convex, and $P(K)$ is the full algebra of continuous functions. If $Q$ has nonzero eigenvalues of the same sign, the polynomial hull of $K$ is described in terms of a foliation by certain simple analytic sets in $C^{2}$, and $P(K)$ is isomorphic to the algebra of continuous functions on the hull whose restriction to the interior of each analytic set is holomorphic.

1. This paper studies the function theoretic properties of the graph of a smooth complex valued function

$$
f(z)=Q(z)+o\left(|z|^{2}\right)
$$

near zero in $C^{2}$, where $Q$ is a real valued quadratic form with no zero eigenvalues and rank $f \leqq 1$ near 0 . The last condition refers to $f$ as a map into $\boldsymbol{R}^{2}$, and simply means that the ordinary Jacobian determinant of $f$ vanishes in a neighborhood of 0 . It is emphasized that the term $o\left(|z|^{2}\right)$ may take complex values. The form $Q$ is elliptic if both eigenvalues have the same sign, and hyperbolic if the signs are different. The latter case is the principal object of study here.

THEOREM 1.1. If $Q$ is hyperbolic and rank $f \leqq 1$ near 0 , there exists a compact neighborhood $D$ of 0 such that any compact subset of the graph

$$
M=\{(z, f(z)): z \in D\}
$$

is polynomially convex. 
The function $f(z)=z^{2}+\bar{z}^{2}=2\left(x^{2}-y^{2}\right)$ provides a simple example. Many others can be constructed from it, as follows. Let $g: \boldsymbol{R} \rightarrow \boldsymbol{C}$ be a smooth function such that $g(0)=0<g^{\prime}(0)$. Then $f(z)=g\left(z^{2}+\bar{z}^{2}\right)$ satisfies Theorem 1.1. In general, however, functions which satisfy the theorem will exhibit a more elaborate structure. An example which, in view of $\S 2$, is "worst possible" is found in [3, Case 2].

Any smooth real two-dimensional submanifold $M$ of $C^{2}$ may, after a local biholomorphic coordinate change due to Bishop [1, p. 5], be represented near a point where its tangent space is complex linear as a graph near 0 of a function having the form (1.1), but in general with no constraint on the eigenvalues. While it is likely [4] that only a relatively few manifolds admit a thin representation (1.1) where rank $f \leqq 1$ near 0 , there are nevertheless enough which do to make positive results for this case interesting.

The conditions on the eigenvalues of $Q$ can be reformulated in a manner that is seen to be invariant under local biholomorphic coordinate changes of $C^{2}$ [1]. However, the rank condition is definitely not such an invariant, as a simple example $[4, \S 1]$ will show. The methods used here, which for technical reasons depend heavily on this condition, are thus not appropriate to a general study of the local function theory of a two manifold in $C^{2}$ near a point where its tangent space is complex linear. However they do yield the result on approximation below, and may also be used where $Q$ is elliptic. Bishop [1] developed the representation (1.1) (in greater generality, applicable to a wider range of dimensions for $M$ and the ambient space) to study the polynomially convex hull of $M$ near an elliptic point. He found that this hull is larger than $M$, and exhibited an analytic structure in it. He also made further conjectures about the structure of such a hull which are verified here in $\S 4$ for elliptic points subject to the rank condition.

Theorem 1.1 and the following result of J. Wermer can be combined to yield a statement on uniform approximation to continuous functions by polynomials in $z$ and $f$. Given a compact set $D$ in $C$ and a continuous function $f: D \rightarrow C$, denote by $[z, f]$ the uniform closure of polynomials (with constant term) in $z$ and $f$. That is, $[z, f]$ is the smallest closed subalgebra with identity of $C(D)$, the Banach algebra of all continuous complex valued functions on $D$.

THEOREM 1.2. (Wermer [10, Appendix]). If $U$ is open in $C$, $f$ : $U \rightarrow C$ is continuously differentiable, and $D \subset U$ is a compact set such that 


$$
\begin{aligned}
& M=\{(z, f(z)): z \in D\} \text { is polynomially convex, and } \\
& E=\{\zeta \in D: \partial f / \partial \bar{z}(\zeta)=0\} \text { has Lebesgue measure zero in } C,
\end{aligned}
$$

then $[z, f]=C(D)$.

Wermer proved this result for the case of a disk $D=\{z:|z| \leqq \rho\}$. Its extension to an arbitrary compact set calls for almost no modification of his proof. Or one can obtain it as a special case of Theorem 4.1 of [5].

A first order example verifying Theorem 1.2 is furnished by $f(z)=\bar{z}$, and a second order one by $f(z)=z^{2}+\bar{z}^{2}$. In fact, the conditions (1.3) and (1.4) are met by any function $f$ which satisfies the hypotheses of Theorem 1.1. For the rank condition means that the Jacobian $|\partial f / \partial z|^{2}-|\partial f / \partial \bar{z}|^{2}$ vanishes in a neighborhood $U$ of 0 . Thus the set $E$ coincides in $U$ with the set where both $\partial f / \partial z$ and $\partial f / \partial \bar{z}$ vanish, and all of these points are critical points for $\operatorname{Re} f$. Since $Q$ has no zero eigenvalues, $\operatorname{Re} f$ has a nondegenerate and hence isolated [9, Cor. 2.3] critical point at 0 . Therefore for sufficiently small $U$, $E \cap U=\{0\}$. According to Theorem 1.1, there is a compact neighborhood $D$ of $0, D \subset U$, satisfying (1.3). This proves the following.

THEOREM 1.3. If $f$ is a smooth function of the form (1.1) with $Q$ hyperbolic and rank $f \leqq 1$ near 0 , then there is a compact neighborhood $D$ of zero such that $[z, f]=C(D)$.

Of course, the same conclusion must still obtain when $D$ is replaced by any compact subset. Results of this type were given by Mergelyan.

Theorem 1.4. (Mergelyan [8, Th. 1.5, p. 27]) If $D$ is a compact disk and $f: D \rightarrow \boldsymbol{R}$ is a continuous real valued function such that for each $\beta \in \boldsymbol{R}$ the level set $L_{\beta}=\{z \in D: f(z)=\beta\}$ has no interior and $\boldsymbol{C}$ $-L_{\beta}$ is connected, then $[z, f]=C(D)$.

Again, $f(z)=z^{2}+\bar{z}^{2}$ is an example. Moreover, if the function of Theorem 1.3 is real valued, one sees easily (c. f. $\S 2$ ) that it satisfies Mergelyan's hypotheses on a sufficiently small disk $D$. Thus for real-valued functions Theorems 1.3 and 1.1 are simple corollaries of Theorem 1.4. However, the usual techniques of proof for Theorem 1.4 depend heavily on the fact that $f$ takes only real values. They do not seem applicable to a complex valued function. The methods developed here do not use Theorem 1.4 or its proof at all, although they do make use of similar features of the level sets of the function 
$f$ of Theorems 1.3 and 1.1. In addition, since $f$ is complex valued, it turns out that certain connectivity properties of its image in $C$ must also be accounted for.

These topological properties are investigated in $\S 2$. The proof of Theorem 1.1 is contained in $\S 3$, and the results for the elliptic case given in $\S 4$. In $\S 5$ are collected some simple examples and a few remarks on the scope of the methods used here.

2. Topological Preliminaries to Theorem 1.1. This section treats some topological properties of the image and certain "level" sets of a function (1.1) in which $Q$ is hyperbolic and rank $f \leqq 1$ on a neighborhood $U$ of 0 . For certain compact sets $D \subset U$, it is desired to identify the bounded components $V$ of $C-f(D)$ and the inverse images $f^{-1}(\bar{V})$ of their closures.

Since $\operatorname{Re} f$ has a nondegenerate critical point at 0 , there exist [9, Lemma 2.2] smooth coordinates $(\xi, \eta)$ for a neighborhood of 0 in which $\operatorname{Re} f=\xi^{2}-\eta^{2}$, so that

$$
f=\xi^{2}-\eta^{2}+o\left(\xi^{2}+\eta^{2}\right),
$$

where the higher order term is purely imaginary. Since the results desired in this section are local and purely topological, it may be assumed that (2.1) holds throughout $U$, and that $U=\left\{(\xi, \eta): \xi^{2}+\eta^{2}<a\right\}$ for some $a>0$.

It will be shown that on $U-\{0\}, \operatorname{Im} f$ is locally a smooth function of $\operatorname{Re} f$. This local information is then pieced together to get a description of $f(U)$ as the union of the graphs of two smooth real valued functions of a real variable, and to obtain information about the level sets of $f$ in $U$.

Now the function $\xi^{2}-\eta^{2}$ is the first coordinate of the map $\left(\xi^{2}-\eta^{2}, 2 \xi \eta\right)\left(\boldsymbol{R}^{2}\right.$ notation for $\left.(\xi+i \eta)^{2}\right)$ whose restriction $\phi$ to the set $U_{1}=\left\{(\xi, \eta): \xi^{2}+\eta^{2}<a\right.$ and $\left.\xi>-\eta\right\}$ is a diffeomorphism of $U_{1}$ with $\left\{(u, v): u^{2}+v^{2}<a^{2}\right.$ and either $u \neq 0$ or $\left.v>0\right\}=U_{1}^{\prime}$, the disk of radius $a$ and center 0 in the $(u, v)$ plane with the negative $v$-axis removed.

This map transports $f$ to $f \circ \phi^{-1}$, where

$$
f \circ \phi^{-1}(u, v)=u+i f^{\prime}(u, v) \text {. }
$$

The function $f^{\prime}$ is real and due to the rank condition $\partial f^{\prime} / \partial v=0$ on $U^{\prime}{ }_{1}$. An elementary argument will show that $f^{\prime}$ is a smooth function $g_{1}$ of $u$ alone on $U_{1}^{\prime}$, and $g_{1}$ is defined on $(-a, a)$. Thus $\operatorname{Im} f=$ 
$f^{\prime} \circ \dot{\phi}=g_{1} \circ \operatorname{Re} f$ on $U_{1}$. In the same way, there exists another smooth function $g_{2}$ on $(-a, a)$ such that $\operatorname{Im} f=g_{2} \circ \operatorname{Re} f$ on $U_{2}=$ $\left\{(\xi, \eta): \xi^{2}+\eta^{2}<a\right.$ and $\left.\xi<-\eta\right\}$. Of course, it can be shown just as easily that $\operatorname{Im} f$ is a smooth function of $\operatorname{Re} f$ in some neighborhood of each point of $U-\{0\}$.

Since $\operatorname{Re} f$ is zero along the curves $Z_{1}=\left\{(\xi, \xi): 0<2 \xi^{2}<a\right\}$ and $z_{2}=\left\{(\xi,-\xi): 0<2 \xi^{2}<a\right\}$, it follows that $f$ is locally constant on these sets, hence constant on each component of $Z_{1} \cup Z_{2}$. But 0 is an accumulation point of each component, and $f(0)=0$. Therefore $f(\xi, \xi)=$ $f(\xi,-\xi)=0$ if $2 \xi^{2}<a$. This shows that $g_{1}(0)=g_{2}(0)=0$ and that $f(U)=$ graph $g_{1} \cup$ graph $g_{2}$. The following result is proved.

Lemma 2.1. If $U, U_{1}$, and $U_{2}$ are defined as above, then

$$
f(\xi, \eta)=0 \text { if and only if } \operatorname{Re} f(\xi, \eta)=0 \text {, and }
$$

(2.4) There exist smooth functions $g_{1}, g_{2}:(-a, a) \rightarrow \boldsymbol{R}$ such that $\operatorname{Im} f=$ $g_{j} \circ \operatorname{Re} f$ on $U_{j}$, and hence $f(U)=$ graph $g_{1} \cup$ graph $g_{2}$.

Thus as a point crosses from $U_{1}$ to $U_{2}$ the image point moves along graph $g_{1}$ through 0 into graph $g_{2}$. An example which illustrates this behavior is found in [3, Case 2] and explained in detail.

Now for any positive number $r<a$ and for $D=\left\{(\xi, \eta): \xi^{2}+\eta^{2} \leqq r\right\}$, it is intuitively clear that the bounded complementary components of $f(D)$ are just the sets bounded by the graphs of $g_{1}$ and $g_{2}$ between two consecutive zeros of $g_{1}-g_{2}$ in $[-r, r]$. This is verified in the following Lemma. It is clear from Lemma 2.1 that $f(D)$ is the union of those parts of the graphs of $g_{1}$ and $g_{2}$ which lie over $[-r, r]$.

LEMMA 2.2. If $V$ is a bounded component of $C-f(D)$ then there exist numbers $b_{1}, b_{2}$ in $[-r, r]$ with $b_{1}<b_{2}$ and such that

$$
\begin{aligned}
& g_{1}\left(b_{1}\right)-g_{2}\left(b_{1}\right)=0=g_{1}\left(b_{2}\right)-g_{2}\left(b_{2}\right) \text { but } g_{1}-g_{2} \text { has no zeros in }\left(b_{1}, b_{2}\right), \text { and } \\
& V=\left\{(u, v): b_{1}<u<b_{2} \text { and } v \text { is between } g_{1}(u) \text { and } g_{2}(u)\right\} .
\end{aligned}
$$

Proof. If $b_{1}<b_{2}$ are consecutive zeros of $g_{1}-g_{2}$ in $[-r, r]$ the set (2.6) is a component of $C-f(D)$. For it is easy to verify that it is both open and closed in $C-f(D)$, and connected.

To complete the proof, it is remarked that each point $(u, v)$ of a nonempty bounded component $V$ of $C-f(D)$ must be such that $-r \leqq u \leqq r$ and $v$ is between $g_{1}(u)$ and $g_{2}(u)$. For if a point does 
not satisfy these conditions, it can clearly be connected by a vertical line in $C-f(D)$ to the unbounded component. Let $b_{1}^{\prime}=\inf \{\operatorname{Re} w: w \in V\}$ and $b_{2}^{\prime}=\sup \{\operatorname{Re} w: w \in V\}$. Then there are no zeros of $g_{1}-g_{2}$ between $b_{1}^{\prime}$ and $b_{2}^{\prime}$. For the existence of a point $b$ with $b_{1}^{\prime}<b<b_{2}^{\prime}$ and $g_{1}(b)=$ $g_{2}(b)=0$ would show, by the remark above, that $V \subset\{w: \operatorname{Re} w<b\} \cup$ $\{w: \operatorname{Re} w>b\}$. But $V$ meets both of these sets by definition of $b_{1}^{\prime}$ and $b_{2}^{\prime}$, and this would contradict its connectivity. Now let $b_{1}$ be the greatest zero of $g_{1}-g_{2}$ occurring at or before $b_{1}^{\prime}$, and $b_{2}$ be the least one which is greater than or equal to $b_{2}^{\prime}$. Then there are no zeros between $b_{1}$ and $b_{2}$ so the set (2.6) defined by $b_{1}$ and $b_{2}$ is a component of $C-f(D)$. Since it contains $V$, this set and $V$ must coincide, which proves the lemma.

3. Proof of Theorem 1.1. With the coordinates $(\xi, \eta)$ and the neighborhood $U$ of 0 chosen in $\S 2$, it will suffice to show that the graph (1.2) determined by $D=\left\{(\xi, \eta): \xi^{2}+\eta^{2} \leqq r\right\}$ is polynomially convex for some $r$ with $a>r>0$. For as the argument following Theorem 1.2 shows, that result is then applicable to $D$ and hence $[z, f]=C(D)$. It follows that every compact subset of (1.2) is polynomially convex.

It must be shown how to choose $D$ so that if $(\alpha, \beta)$ is a point in $C^{2}$ such that

$$
|p(\alpha, \beta)| \leqq \sup _{M}|p|
$$

for every polynomial $p$ in two variables, then $(\alpha, \beta) \in M$.

For a given $D$, it follows from (3.1) that the map $p \rightarrow p(\alpha, \beta)$ extends to an algebra homomorphism $\lambda$ of $P(M)$ onto $C$, where $P(M)$ is the uniform closure on $M$ of all polynomials. There is a probability measure $\mu$ on $M$ [6, pp. 31-2] which represents $\lambda$ in the sense that

$$
\lambda(g)=\int g d \mu
$$

for each $g$ in $P(M)$. Now if $[z, f]=C(D)$, it is well known [6] that $(\alpha, \beta) \in M$ and further that $\mu$ is a unit point mass at $(\alpha, \beta)$. In a rough sense, the proof below shows that the converse is true for the functions considered here. It is reasoned that $(\alpha, \beta) \in M$ by locating the support of $\mu$ in a sufficiently small and well behaved set. The argument makes use of the fact that the action of $\lambda$ depends only on the behavior of functions on support $\mu$.

The support of $\mu$ will be located through its relations (3.2) and 
(3.3) with the supports of the measures $\mu_{1}$ and $\mu_{2}$ induced from $\mu$ on the $z$ - and $w$-planes respectively by the coordinate projections $\pi_{1}:(z, w) \rightarrow z$ and $\pi_{2}:(z, w) \rightarrow w$. For all Borel sets $E, \mu_{j}(E)=$ $\mu\left(\pi_{j}^{-1}(E)\right), j=1,2$. It is straightforward to verify that

$$
\text { support } \mu \subset \pi_{j}^{-1} \text { (support } \mu_{j} \text { ) , }
$$

and

$$
\pi_{j}(\operatorname{support} \mu) \supset \text { support } \mu_{j} \text {. }
$$

Another well-known property is that

$$
\int g d \mu_{j}=\int\left(g \circ \pi_{j}\right) d \mu
$$

for all measurable functions $g$.

Now from (3.3) support $\mu_{2} \subset f(D)$. Also, for any polynomial $p_{2}$ in $w$, it follows from (3.4) that

$$
p_{2}(\beta)=\left(p_{2} \circ \pi_{2}\right)(\alpha, \beta)=\lambda\left(p_{2} \circ \pi_{2}\right)=\int p_{2} d \mu_{2} .
$$

These two facts imply that

$$
\left|p_{2}(\beta)\right| \leqq \sup _{f(D)}\left|p_{2}\right|
$$

It follows from (3.6) and Runge's theorem that $\beta$ must belong to the union $E$ of $f(D)$ and its bounded complementary components in the $w$-plane.

Of course, it is desired to show that $\beta \in f(D)$, but at this point it may very well belong to some bounded component $V$ of $C-f(D)$. In any case, $\beta$ certainly belongs to $\bar{V}$ for some such $V$, since $f(D)$ has no interior (Lemma 2.1 or Sard's theorem).

So far it is shown that $\mu_{2}$ is a probability measure supported on $f(D)=\partial E$ which according to (3.5) represents evaluation at $\beta$ on polynomials $p_{2}$ in $w$.

But with an independent argument $[2$, p. 80] using elementary functional analysis and the ordinary maximum principle, one can find a probability measure $\sigma$ supported on $\partial V$ which also represents evaluation at $\beta$. This and (3.5) show that $\mu_{2}-\sigma$ is a measure supported on $f(D)=\partial E$ which annihilates polynomials in $w$. Since $\mu_{2}-\sigma$ is a real measure, it annihilates their real parts as well. Now it is well known [2, Th. 3.4.14] that because $C-E$ is connected, the space of real parts of polynomials in $w$ is uniformly dense in the space of continuous real functions on $\partial E$. Therefore $\mu_{2}-\sigma=0$, proving that 
support $\mu_{2} \subset \partial V$.

It follows from (3.2), (3.3), and Lemma 2.2 that support $\mu_{1} \subset$ $f^{-1}(\partial V)=\left\{(\xi, \eta): b_{1} \leqq \xi^{2}-\eta^{2} \leqq b_{2}\right\}$. Now regard $f^{-1}(\partial V)$ as $L_{1} \cup L_{2}$, where $L_{1}=\left\{(\xi, \eta) \in f^{-1}(\partial V): \xi \geqq-\eta\right\}$ and $L_{2}=\left\{(\xi, \eta) \in f^{-1}(\partial V): \xi \leqq-\eta\right\}$. To complete the proof, it will be necessary to assure that for any $V, L_{1}$ and $L_{2}$ are disjoint. This will be the case when $0 \notin\left[b_{1}, b_{2}\right]$ for any two consecutive zeros $b_{1}$ and $b_{2}$, which amounts to requiring that neither can ever be zero.

This is already true if 0 in the $w$-plane is an accumulation point of the zeros of $g_{1}-g_{2}$ both from the right and from the left. But if, say, 0 is not an accumulation point of zeros from the right, then $D$ may be shrunk so that $g_{1}-g_{2}$ has no zeros with positive real part. For such $D$ there is no $b_{2}>0$, hence no $b_{1}=0$. A further shrinkage of $D$ will take care of the case where 0 is not an accumulation point from the left. Thus it can always be achieved that $L_{1} \cap L_{2}=\phi$ for any bounded component $V$.

A calculation like (3.5) will show that

$$
p_{1}(\alpha)=\int p_{1} d \mu_{1}
$$

for any polynomial $p_{1}$ in $z$, so that

$$
\left|p_{1}(\alpha)\right| \leqq \sup _{L_{1} \cup L_{2}}\left|p_{1}\right|
$$

Since $C-L_{1} \cup L_{2}$ is connected, it follows that $\alpha \in L_{1} \cup L_{2}$. Assume $\alpha \in L_{1}$. Then since $L_{1} \cap L_{2}=\phi$, the characteristic function $\chi$ of $L_{1}$ relative to $L_{1} \cup L_{2}$ can be approximated uniformly on $L_{1} \cup L_{2}$ by polynomials in $z$ (Runge's theorem again). Therefore by taking limits in (3.7) one finds that

$$
1=\chi(\alpha)=\int_{L_{1} \cup L_{2}} \chi d \mu_{1}=\mu_{1}\left(L_{1}\right)
$$

Since $\mu_{1}$ is clearly a probability measure, it follows that support $\mu_{1} \subset L_{1}$.

By means of (3.2) and (3.4) this implies that support $\mu_{2} \subset f\left(L_{1}\right)=$ graph $\left(g_{1} \mid\left[b_{1}, b_{2}\right]\right)$. Exploiting (3.5) again gives

$$
\left|p_{2}(\beta)\right| \leqq \sup _{f\left(L_{1}\right)}\left|p_{2}\right|
$$

for all polynomials $p_{2}$ in $w$. Since $\boldsymbol{C}-f\left(L_{1}\right)$ is connected, this shows that $\beta \in f\left(L_{1}\right)$. Thus the unit point mass at $\beta$ is another probability measure on $f(D)$ also representing evaluation at $\beta$, from which follows as before that support $\mu_{2}=\{\beta\}$. Hence support $\mu_{1} \subset f^{-1}(\beta)$. But $f^{-1}(\beta)$ 
is polynomially convex in the $z$-plane. It follows from the same calculation as (3.8) with the supremum extended only over $f^{-1}(\beta)$, that $\alpha \in f^{-1}(\beta)$. This proves Theorem 1.1.

4. The polynomial hull and approximation near a thin elliptic point. The same technique can be used to determine the polynomially convex hull of a small disk in a thin manifold near an elliptic point. In this case the details are simpler.

It turns out that the image under $f$ of a certain small coordinate disk $D$ is the graph of a single function, which graph can be regarded as the domain of a parameter indexing a family of analytically imbedded disks in $C^{2}$ which exhaust the hull of the graph $M$ of $f$ over $D$. A simple example of this occurs when the term $o\left(|z|^{2}\right)$ in (1.1) is identically zero. Then $f=Q$ and its graph over any closed disk $D$ may be visualized accurately in $\boldsymbol{R}^{3}$ (imbedded in $\boldsymbol{C}^{2}$ as the set $\{\operatorname{Im} w=0\}$ ) as a simple "cup shaped" quadric surface, whose polynomially convex hull is obtained by filling the cup in $\boldsymbol{R}^{3}$. For the cup is filled by the analytic disks cut out by $Q$ from planes parallel to the $z$-plane, and the ordinary maximum principle applied to each polynomial $p(z, w)$ as a function of $z$ for fixed $w$ will show that each such disk is in the hull. Since the set obtained by thus filling the cup is geometrically convex, it is polynomially convex, and hence coincides with the hull of $M$. The same results occur in general when the graph $M$ is "bent out" into $C^{2}$ by the term $o\left(|z|^{2}\right)$ (while retaining the rank condition).

Now $Q$ has two eigenvalues of the same sign, and no generality is lost by taking them both positive. The function $\operatorname{Re} f$ again has a nondegenerate critical point at 0 , so it follows as in $\S 2$ that there exist coordinates $(\xi, \eta)$ in a neighborhood $U$ of 0 such that

$$
f=\xi^{2}+\eta^{2}+o\left(\xi^{2}+\eta^{2}\right)
$$

where the higher order term is imaginary. It may again be assumed that $U=\left\{(\xi, \eta): \xi^{2}+\eta^{2}<a\right\}$ for some $a>0$ and that (4.1) holds throughout $U$.

It is possible to compute the hull of any compact subset of the graph $\{(z, f(z)): z \in U\}$. Any such set is clearly of the form $M_{K}=$ $\{(z, f(z)): z \in K\}$ for some compact subset $K$ of $U$. For a given $K$ and $\beta \in \boldsymbol{C}$, let

$$
L_{\beta}=\{z \in K: f(z)=\beta\}
$$


As the intersection of $K$ with a closed level set of $f, L_{\beta}$ is clearly compact. If it has a bounded complementary component $V$, and $p$ is a polynomial in two variables, then the maximum principle applied to $z \rightarrow p(z, \beta)$ shows teat $V \times\{\beta\} \subset \widehat{M_{K}}$, the polynomially convex hull of $M_{K}$. Thus if $\widehat{L_{\beta}}$ denotes the union of $L_{\beta}$ and its bounded complementary components, it is clear that

$$
\widehat{M_{K}} \supset \bigcup_{\beta \in C}\left(\widehat{L_{\beta}} \times\{\beta\}\right)
$$

Moreover, if $A_{K}=\left\{f \in C\left(\widehat{M_{K}}\right): f \mid \widehat{L_{\beta}} \times\{\beta\}\right.$ is holomorphic in $z$ on interior $\left.\widehat{L_{\beta}}\right\}$, then $p\left(\widehat{M_{K}}\right) \subset \mathrm{A}_{K}$ by elementary considerations.

THEOREM 4.1. With the definitions above,

$$
\widehat{M_{K}}=\bigcup_{\beta \in C}\left(\widehat{L_{\beta}} \times\{\beta\}\right),
$$

and

$$
P\left(\widehat{M}_{K}\right)=A_{K}
$$

Of course, (4.3) gives a description of $P\left(M_{K}\right)$ as well, since it is always [6, p. 67] naturally isomorphic to $P\left(\widehat{M}_{K}\right)$. It is easy to see that (4.3) is true in the example above with $o\left(|z|^{2}\right)=0$ if $K$ is a closed disk concentric with the origin.

Proof. At each point $z$ of $U-\{0\}, u=\operatorname{Re} f$ is the first coordinate of a diffeomorphism $\phi=(u, v)$ of some open neighborhood $U_{z}$ of $z$ onto an open set $U_{z}^{\prime}$ in $\boldsymbol{R}^{2}$. As before, this map transports $f$ to $f \circ \phi^{-1}$ described by (2.2) on $U_{z}^{\prime}$ and the rank condition applies again to $f^{\prime}$, showing that near $z, \operatorname{Im} f$ is a smooth function of $\operatorname{Re} f$. In particular, $\operatorname{Im} f$ is locally constant on each level set

$$
\{z \in U: f(z)=\beta\} \text {. }
$$

Since each such set is (either empty or) a circle in the $(\xi, \eta)$ coordinates, it is connected. Thus $\operatorname{Im} f$ is constant on each set (4.4), which shows that there exists a smooth function $g:(0, a) \rightarrow \boldsymbol{R}$ such that $\operatorname{Im} f=g \circ \operatorname{Re} f$ on $U-\{0\}$. Since $f(0)=0$ it follows from continuity of $f$ that $g(u) \rightarrow 0$ as $u \rightarrow 0$. Letting $g(0)=0, g$ is smooth on $[0, a)$ and $f(U)$ is the graph of $g$ on $[0, a)$.

With this description of $f(U)$ it is possible to verify (4.2) just as in Theorem 1.1. Let $(\alpha, \beta) \in \widehat{M}_{K}$, so that 


$$
|p(\alpha, \beta)| \leqq \sup \left\{|p(z, w)|:(z, w) \in M_{K}\right\}
$$

for every polynomial $p$. There is a probability measure $\mu$ on $M_{K}$ representing $(\alpha, \beta)$ as before, so that

$$
\left|p_{2}(\beta)\right| \leqq \sup _{f(K)}\left|p_{2}\right|
$$

whenever $p_{2}$ is a polynomial in $w$. Now as a compact subset of the graph of $g, f(K)$ is polynomially convex in the $w$-plane, so $\beta \in f(K)$. Therefore support $\mu_{2}=\{\beta\}$, so support $\mu \subset L_{\beta} \times\{\beta\}$ and support $\mu_{1} \subset L_{\beta}$. This shows that

$$
\left|p_{1}(\alpha)\right| \leqq \sup \left\{\left|p_{1}(z)\right|: z \in L_{\beta}\right\}
$$

for all polynomials $p_{1}$ in $z$. Therefore $\alpha \in \widehat{L_{\beta}}$, or $(\alpha, \beta) \in \widehat{L_{\beta}} \times\{\beta\}$, proving (4.2).

The proof of (4.3) will use the general Stone-Weierstrass Theorem of Bishop [2, Th. 2.7.5] or [6, p. 60], for which must be located the maximal sets of antisymmetry of $P\left(\widehat{M_{K}}\right)$. Note that Re $w \in P(f(K))$ by Lavrentiev's theorem [6, p. 48], since $f(K)$ is polynomially convex and without interior. Tnus $\operatorname{Re} w \in P\left(\widehat{M_{K}}\right)$, which shows that any maximal set of antisymmetry of this algebra is contained in some $\widehat{L_{\beta}} \times\{\beta\}$. It remains to show that for each $\beta$, a given function $h$ in $A_{K}$ agrees on $\widehat{L_{\beta}} \times\{\beta\}$ with some function in $P\left(\widehat{M}_{K}\right)$. But Mergelyan's theorem [6. p. 48] shows that $h$ may be approximated uniformly on $\widehat{L_{\beta}} \times\{\beta\}$ by polynomials in $z$. Since $P\left(\widehat{M_{K}}\right)$ restricted to any maximal set of antisymmetry is closed [2], [6], this completes the proof.

If $K=\left\{(\xi, \eta): \xi^{2}+\eta^{2} \leqq r\right\}$, then for each $\beta \in$ graph $g, L_{\beta}=\left\{(\xi, \eta): \xi^{2}+\right.$ $\left.\eta^{2}=\operatorname{Re} \beta\right\}$, so $\widehat{L_{\beta}}=\left\{(\xi, \eta): \xi^{2}+\eta^{2} \leqq \operatorname{Re} \beta\right\}$.

Corollary. Let $0 \leqq r<a$ and $K=\left\{(\xi, \eta): \xi^{2}+\eta^{2} \leqq r\right\}$. Then

$$
\begin{aligned}
\widehat{M_{K}} & =\bigcup_{0 \leqq t \leqq r}\left\{(\xi, \eta): \xi^{2}+\eta^{2} \leqq t\right\} \times\{t+i g(t)\} \\
& =\bigcup_{\beta \in f(K)}\left\{(\xi, \eta): \xi^{2}+\eta^{2} \leqq \operatorname{Re} \beta\right\} \times\{\beta\} \\
& =\{(\alpha, \beta): \alpha \in K, \beta \in f(K), \text { and } \operatorname{Re} f(\alpha) \leqq \operatorname{Re} \beta\},
\end{aligned}
$$

and $P\left(\widehat{M}_{K}\right)=\left\{h \in C\left(\widehat{M}_{K}\right)\right.$ : for each $\beta \in f(K), z \rightarrow h(z, \beta)$ is holomorphic where $\left.\xi^{2}+\eta^{2}<\operatorname{Re} \beta\right\}$.

The first and second expressions for $\widehat{M_{K}}$ exhibit a foliation of it 
by analytic disks parallel to the $z$-plane. In this special case the conjectures of Bishop [1. p. 12] about the hull near an elliptic point are verified.

It should be remarked that for such $K$, the rational hull $[6, p .69]$ of $M_{K}$ is the same as $\widehat{M}_{K}$ (and no smaller). In fact the spectrum of the inductive limit algebra $H\left(M_{K}\right)$ [7] is still $\widehat{M}_{K}$. These facts follow from a straightforward argument based on the Kontinuitätsatz applied at 0 .

5. Remarks. The "Parabolic" case where $Q$ has one or more zero eigenvalues is indeterminate by purely second order methods. For example $f_{1}(\xi, \eta)=\xi^{2}-\eta^{4}$ and $f_{2}(\xi, \eta)=\xi^{2}+\eta^{4}$ have the same second order properties, yet one sees by the methods used here that over any compact disk $D$ centered at 0 the graph of $f_{1}$ is polynomially convex while that of $f_{2}$ is not.

It is not difficult to formulate other conditions under which the techniques used in Theorems 1.1 and 4.1 will yield similar results. For instance, one could simply assume that $f$ has all the topological properties needed to make the proofs work. Thus one can posit the conclusions of Lemmas 2.1 and 2.2, assume appropriate topological features of the levels sets of $f$, and retain the conclusion of Theorem 1.1.

However, it is difficult to conceive of situations other than those treated here in which these conditions occur together. Moreover, they are obtained here with reasonable economy of hypotheses and in a relatively natural manner.

Another reason the techniques here are not elaborated is that at present it is felt that Theorem 1.1 and consequently Theorem 1.3 are true without the rank condition. Since relaxing this condition will generally entail the loss of the topological prerequisites to the present proof (they are no longer satisfied by the examples below), some other way will have to be found to prove these results without the rank condition.

The following simple examples verify the conclusions of Theorems 1.1 and 1.3 , but do not satisfy the rank condition:

$$
f(z)=z^{2}+\bar{z}^{2}+z^{3}
$$

which the biholomorhic map $(z, w) \rightarrow\left(z, w-z^{3}\right)$ converts to one satisfying these theorems, 


$$
f(z)=z^{2}+\bar{z}^{2}+\bar{z}^{3}
$$

(the map $(z, w) \rightarrow\left(z, w+z^{3}\right)$ does the same thing), and

$$
f(z)=z^{2}+\bar{z}^{2}+z \bar{z}^{2}
$$

(here the map is $\left.(z, w) \rightarrow\left(z, w-z w+z^{3}\right)\right)$. On the other hand, it is unknown if these theorems are verified by such a simple case as

$$
f(z)=z^{2}+\bar{z}^{2}+z^{2} \bar{z}
$$

(for which it seems unlikely [4, Sec. 3] that there exists a non-constant real-valued analytic function of $z$ and $f$ ).

\section{REFERENCES}

1. E. Bishop, Differentiable Manifolds in complex Euclidean space, Duke Math. J., 32 (1965), 1-22.

2. A. Browder, Introduction to Function Algebras, W. A. Benjamin, Inc., New York (1969).

3. M. Freeman, A differential version of a theorem of Mergelyan, To appear in Proceedings of the Park City Symposium on Applications of Several Complex Variables to Analysis, Lecture Notes in Math. 184 Springer-Verlag, Berlin (1971), 37-49.

4. - Local holomorphic convexity of a two-manifold in $\boldsymbol{C}^{2}$, Rice University Studies, 56 (Spring, 1970), 171-180.

5. - Some Conditions for Uniform Approximation on a Manifold, Function Algebras, Scott, Foresman and Co., Chicago (1966), 42-60.

6, T. W. Gamelin, Uniform Algebras, Prentice-Hall. Inc., Englewood Cliffs, N. J. (1969).

7. R. Harvey and R. O. Wells, Jr., Compact holomorphically convex subsets of a Stein manifold, Trans. Amer. Math. Soc., 136 (1969), 509-516.

8. S. N. Mergelyan, Uniform approximation to functions of a complex variable. Amer. Math. Soc. Translation 101 (1954).

9. J. Milnor, Morse theory, Ann. Math. Studies 51, Princeton, N. J. (1963).

10. J. Wermer, Polynomially convex disks, Math. Ann. 158 (1965), 6-10.

Received December 4, 1970, and in revised form March 29, 1971. This work was supported by NSF Grant GP-8997.

RICE UNIVERSITY

AND

UNIVERSITY OF KENTUCKY 



\section{PACIFIC JOURNAL OF MATHEMATICS}

\section{EDITORS}

\author{
H. SAMELSON \\ Stanford University \\ Stanford, California 94305 \\ C. R. HовBY \\ University of Washington \\ Seattle, Washington 98105
}

J. DUGUndis

Department of Mathematics

University of Southern California

Los Angeles, California 90007

RICHARD ARENS

University of California

Los Angeles, California 90024

\section{ASSOCIATE EDITORS}
E. F. BeCKenbaCH
B. H. NeUmanN
F. WOLF
K. YoshidA

\section{SUPPORTING INSTITUTIONS}

\author{
UNIVERSITY OF BRITISH COLUMBIA \\ CALIFORNIA INSTITUTE OF TECHNOLOGY \\ UNIVERSITY OF CALIFORNIA \\ MONTANA STATE UNIVERSITY \\ UNIVERSITY OF NEVADA \\ NEW MEXICO STATE UNIVERSITY \\ OREGON STATE UNIVERSITY \\ UNIVERSITY OF OREGON \\ OSAKA UNIVERSITY \\ UNIVERSITY OF SOUTHERN CALIFORNIA
}

\author{
STANFORD UNIVERSITY \\ UNIVERSITY OF TOKYO \\ UNIVERSITY OF UTAH \\ WASHINGTON STATE UNIVERSITY \\ UNIVERSITY OF WASHINGTON

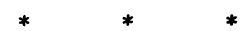 \\ AMERICAN MATHEMATICAL SOCIETY \\ CHEVRON RESEARCH CORPORATION \\ NAVAL WEAPONS CENTER
}

The Supporting Institutions listed above contribute to the cost of publication of this Journal, but they are not owners or publishers and have no responsibility for its content or policies.

Mathematical papers intended for publication in the Pacific Journal of Mathematics should be in typed form or offset-reproduced, (not dittoed), double spaced with large margins. Underline Greek letters in red, German in green, and script in blue. The first paragraph or two must be capable of being used separately as a synopsis of the entire paper. The editorial "we" must not be used in the synopsis, and items of the bibliography should not be cited there unless absolutely necessary, in which case they must be identified by author and Journal, rather than by item number. Manuscripts, in duplicate if possible, may be sent to any one of the four editors. Please classify according to the scheme of Math. Rev. Index to Vol. 39. All other communications to the editors should be addressed to the managing editor, Richard Arens, University of California, Los Angeles, California, 90024.

50 reprints are provided free for each article; additional copies may be obtained at cost in multiples of 50 .

The Pacific Journal of Mathematics is published monthly. Effective with Volume 16 the price per volume (3 numbers) is $\$ 8.00$; single issues, $\$ 3.00$. Special price for current issues to individual faculty members of supporting institutions and to individual members of the American Mathematical Society: $\$ 4.00$ per volume; single issues $\$ 1.50$. Back numbers are available.

Subscriptions, orders for back numbers, and changes of address should be sent to Pacific Journal of Mathematics, 103 Highland Boulevard, Berkeley, California, 94708.

PUBLISHED BY PACIFIC JOURNAL OF MATHEMATICS, A NON-PROFIT CORPORATION

Printed at Kokusai Bunken Insatsusha (International Academic Printing Co., Ltd.), 7-17, Fujimi 2-chome, Chiyoda-ku, Tokyo, Japan. 


\section{Pacific Journal of Mathematics}

Vol. 38, No. $2 \quad$ April, 1971

Richard Davis Anderson and Thomas Ashland Chapman, Extending

homeomorphisms to Hilbert cube manifolds .................. 281

Nguyen Huu Anh, Restriction of the principal series of $\operatorname{SL}(n, \mathbf{C})$ to some

reductive subgroups................................ 295

David W. Boyd, Indices for the Orlicz spaces . . . . . . . . . . . . 315

William Garfield Bridges, The polynomial of a non-regular digraph ...... 325

Billie Chandler Carlson, Robert K. Meany and Stuart Alan Nelson, Mixed

arithmetic and geometric means........................ 343

H. A. Çelik, Commutative associative rings and anti-flexible rings ...... 351

Hsin Chu, On the structure of almost periodic transformation groups ...... 359

David Allyn Drake, The translation groups of n-uniform translation

Hjelmslev planes ................................ 365

Michael Benton Freeman, The polynomial hull of a thin two-manifold . . . . 377

Anthony Alfred Gioia and Donald Goldsmith, Convolutions of arithmetic

functions over cohesive basic sequences .................... 391

Leslie C. Glaser, A proof of the most general polyhedral Schoenflies

conjecture possible ................................

Thomas Lee Hayden and Ted Joe Suffridge, Biholomorphic maps in Hilbert

space have a fixed point ................................ 419

Roger Alan Horn, Schlicht mappings and infinitely divisible kernels ...... 423

Norman Ray Howes, On completeness ...................... 431

Hideo Imai, Sario potentials on Riemannian spaces................ 441

A. A. Iskander, Subalgebra systems of powers of partial universal

algebras.

Barry E. Johnson, Norms of derivations of $\mathscr{L}(\mathrm{X})$.

David Clifford Kay and Eugene W. Womble, Axiomatic convexity theory and relationships between the Carathéodory, Helly, and Radon numbers

Constantine G. Lascarides, A study of certain sequence spaces of Maddox

and a generalization of a theorem of Iyer .............

C. N. Linden, On Blaschke products of restricted growth .

John S. Lowndes, Some triple integral equations ................. 515

Declan McCartan, Bicontinuous preordered topological spaces ......... 523

S. Moedomo and J. Jerry Uhl, Jr., Radon-Nikodým theorems for the Bochner and Pettis integrals ...

Calvin Cooper Moore and Joseph Albert Wolf, Totally real representations

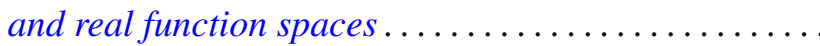

Reese Trego Prosser, A form of the moment problem for Lie groups. ... 